\title{
Highly-Sensitive C-reactive Protein Level and its Association with Intermediate and High Syntax Score in cases of Acute Coronary Syndrome
}

Abdelaziz Rezk, Mohamed Sarhan, Ahmed Elmoghl

Cardiology Department, Faculty of Medicine, Al-Azhar University

\begin{abstract}
Background: Highly-Sensitive C-reactive Protein (hs-CRP) levels are associated with short- and long-term mortality in patients with acute coronary syndrome (ACS). Aim: We investigated if baseline hs-CRP levels are correlated with burden of coronary atherosclerosis assessed by SYNTAX score (SXScore).

Subjects and methods: We investigated 200 patients with ACS who underwent coronary angiography. The patients were divided into groups based on their SXScore: low SXScore $(\leq 22)$, and intermediate-high SXScore $(\geq$ 23). Results: Patients in the intermediate-high SXScore group had higher serum hs-CRP levels in comparison to low SXScore group $(4.61 \pm 1.54 \mathrm{mg} / \mathbf{d L}$ versus $2.37 \pm 0.66 \mathrm{mg} / \mathbf{d L}, P<0.001)$. The mean age of patients and prevalence of diabetes in the intermediate-high SXScore group were statistically insignificantly than in the low SXScore group. Left ventricular ejection fraction (LVEF) was significantly lower in the patients with an intermediate-high SXScore (41 $\pm 9 \%$ ) compared to low SXScore tertiles $(\mathbf{5 0 \pm 8} \%)(\mathbf{P}<\mathbf{0 . 0 0 1})$.

There was significant correlation between the hs-crp levels and the syntax score. The correlation coefficient $(\mathrm{r})=$

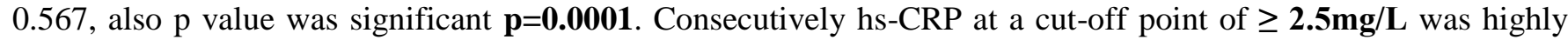
sensitive (97\%) and specific (41\%) and was a strongly independent predictor of a high SXScore in patients with ACS. Where accuracy of test was $(\mathrm{acc}=0.94)$. Conclusion: It is believed that serum levels of hs-CRP on admission in patients with ACS thought to be a strong predictor of the severity and complexity of coronary atherosclerosis together with multivessel disease, and LVEF.
\end{abstract}

Keywords: Inflammation, Severity of atherosclerosis, Coronary artery disease.

\section{INTRODUCTION}

The SYNTAX score (SXScore) is a useful angiographic grading tool and it can be used to accurately quantify the extent, severity, and complexity of coronary artery disease (CAD) ${ }^{(1-3)}$.

The SXScore is an independent predictor of longterm mortality and morbidity in several patient types including acute coronary syndrome (ACS) ${ }^{(4-8)}$.

It is known that important role in the atherosclerotic process performed through inflammation where, it begins as endothelial dysfunction then continues through all stages of plaque formation until its detrimental clinical ischemic complications ${ }^{(9,10)}$. High sensitivity C-reactive protein (hs-CRP), is a well-known acute phase protein that when elevated is considered an extremely sensitive marker of systemic inflammation, is also an important risk factor for atherosclerosis and CAD ${ }^{(6,7)}$.

Many studies showed that hs-CRP levels were a useful marker to predict further cardiovascular events in patients with $\mathrm{CAD}^{(\mathbf{1 1}, \mathbf{1 2})}$. In this study, we aimed to investigate whether hs-CRP was associated with the SXScore in patients with ACS.

\section{METHODS}

\section{Study population:}

We selected 200 patients with ACS who underwent coronary angiography between September 2017 and June 2018. The clinical spectrum of ACS consisted of ST elevation myocardial infarction (STEMI, $\mathrm{n}=117$, $58.5 \%$ ) and non-ST elevation acute coronary syndromes [NST-ACS, including non ST elevation myocardial infarction (NSTEMI) or unstable angina pectoris (USAP), $\mathrm{n}=83,41.5 \%]$, which diagnosed using electrocardiography (ECG) changes and the development of myocardial necrosis. Patients with ACS, an SXScore of zero, history of prior coronary artery bypass grafting surgery (CABG), cardiopulmonary resuscitation before admission, presence of advanced valve disease and advanced renal/ hepatic disease, known malignancy, a history of congestive heart failure, and/or the presence of congestive heart failure ( $\geq$ Killip class III) on admission were excluded from the study.

\section{Diagnostic criteria for STEMI and NSTE-ACS:}

The main symptom of ACS is typical ischemic chest pain. Subjects with ST segment elevation in $\geq 2$ consecutive leads (with the cut-off point being $>0.2$ $\mathrm{mV}$ in leads $\mathrm{V} 1, \mathrm{~V} 2$, or $\mathrm{V} 3$, and $>0.1 \mathrm{mV}$ in the other leads) were diagnosed as STEMI. On other side the diagnosis of NSTE-ACS was a rule-out diagnosis based on the ECG, i.e., lack of persistent ST elevation. Biomarkers (troponins) further distinguish NSTEMI and USAP. Imaging modalities were used to rule out or rule in differential diagnoses. The clinical presentation of NSTE-ACS includes variety of symptoms. Most commonly clinical presentations were described as: Prolonged (> 20 minutes) angina pain at rest; new onset (de novo) angina (Class II or III of the Classification of the Canadian Cardiovascular Society); recent destabilization of previously stable angina with 
at least Canadian Cardiovascular Society Class III angina characteristics (crescendo angina); or post-MI angina. The characteristic ECG changes of NSTEACS are ST-segment depression or transient elevation and/or T-wave change. Furthermore, the diagnosis of NSTE-ACS, based on the measurement of troponins, was further categorized as NSTEMI or USAP. Patients with normal troponin levels were approved as USAP. Patients with high troponin levels were approved as NSTEMI ${ }^{(3)}$.

The study protocol was approved by the local ethics committee and all patients gave their written informed consent.

hs-CRP analysis: Serum hs-CRP levels were measured from blood samples taken at admission to the cardiac care unit or catheter laboratory. Creatinine clearance was also calculated from plasma creatinine levels assessed from blood samples taken at about one hour after first contact ${ }^{(13)}$.

\section{Coronary angiography and SXScore analysis:}

Coronary angiography was done by the Judkins technique. The severity and complexity of coronary atherosclerosis was measured in several ways. The first was a simple classification in number of diseased vessel scoring system. The number of diseased vessels with $\geq 50 \%$ luminal stenosis in major coronary arteries was scored as 1 to 3 diseased vessels. In addition, angiograms were scored according to the SXScore system $^{(2,3)}$. All lesions causing $\geq 50 \%$ narrowing in a coronary artery with a diameter $\geq 1.5 \mathrm{~mm}$ were included in the SXScore calculation. For the calculation, the software on the website (http://www.syntaxscore.com) was used. SXscore was used by 2 interventional cardiologists blinded to the study protocol and patient characteristics and apart from each other. If there was contradiction between the two results, the opinion of a senior interventional cardiologist was applied and a common consensus was obtained. The patients were categorized into groups according to the SXScore: low SXScore $(\leq 22)$, and intermediate-high SXScore $(\geq 23)^{(2-3)}$.

Transthoracic echocardiography (TTE) was done for all patients within 48 hours after admission (Vivid 5; GE Medical System, Horten, Norway). Using the biplane Simpson method left ventricular ejection fraction (LVEF) was measurement

\section{Statistical analysis}

All statistics were done through using SPSS for Windows (version 18.0, SPSS, Chicago, Illinois, USA). Quantitative variables are presented as the mean value \pm SD for parametric variables, and median and minimum-maximum levels for non-parametric variables. Comparisons of parametric values among groups were performed by the Student $\mathrm{t}$ test. Categorical variables were compared with the chi- square test. A two-tailed $\mathrm{P}<0.05$ was considered significant. Univariate logistic regression was used to identify independent predictors of a high SXScore. Receiver operating characteristic (ROC) curve analysis was performed in order to determine the best cutoff value of hs-CRP and the sensitivity and specificity at that point were obtained for predicting a high SXSscore groups.

\section{RESULTS}

A total number of 200 patients met the inclusion and exclusion criteria. The baseline characteristics of the study population classified according to SXScore groups are presented in table 1 . Where no significant differences were present in both groups regarding age, sex, smoking, hypertension and diabetes.

Additionally, LVEF was significantly lower in the patients with an intermediate-high SXScore (41.9) \% compared to low SXScore tertiles 50 $\pm 8 \%(\mathrm{P}<0.001)$. Patients with an intermediate-high SXScore more commonly presented with STEMI, while patients with low tertile more commonly presented with NSTEACS. Laboratory measurements of the patients stratified into SXScore groups are shown in table 2. Subjects in the intermediate-high SXScore group had higher serum hs CRP compared to the low SXScore group (mean hs-CRP levels were $4.61 \pm 1.54 \mathrm{mg} / \mathrm{L}$ versus $2.37 \pm 0.66 \mathrm{mg} / \mathrm{L}, \mathrm{P}<0.001)$. We found a significant relationship between hs-CRP and SXScore with correlation analysis $(\mathrm{r}=0.567, \mathrm{P}<0.001)$ as shown in table 4.

Angiographic characteristics, of patients according to SXScore tertiles are presented in table 3. Patients with an intermediate-high SXScore had a significantly higher rate of multivessel disease and compared to low SXScore patients ( $\mathrm{P}<0.001$ for both). Single, two, and three vessel diseases were detected in $55(55 \%), 31$ (31\%), and $14(14 \%)$, respectively, in low SXScore patients and 26 (26\%), 33 (33\%), and 4 (41\%), respectively, in intermediate-high SXScore patients $(\mathrm{P}$ $<0.001)$. The left anterior descending artery was more commonly the culprit vessel in the intermediate-high SXScore tertile and the right coronary artery was more commonly the culprit vessel in the low SXScore tertile.

Univariate logistic regression analysis was used to estimate if hs-crp could be an independent predictors of intermediate-high SXScore tertile. In this study regarding hs-crp the coefficient of correlation (R) was 0.563 , while coefficient of determination ( $R$ Square) was 0.316 with efficacy of this test (f) is $91.44 \%$, and the $\mathrm{p}$ value for this test was found to be statistically significant table (5).

ROC curve analysis showed that hs-CRP at a cut-off point of $2.5 \mathrm{mg} / \mathrm{L}$ was highly sensitive (97\%) and specific (41\%) and was a strongly independent predictor of a high SXScore in patients with ACS $(\mathrm{AUC}=0.945, \mathrm{P}<0.001)$. (Table 6 -Figure 7$)$. 
Table 1: Basic demographic characteristics of two groups.

\begin{tabular}{|c|c|c|c|c|}
\hline \multicolumn{2}{|c|}{ Variables } & $\begin{array}{c}\text { Intermediate to } \\
\text { High Syntax Score Group } \\
\mathrm{N}=\mathbf{1 0 0} \\
\text { Mean } \pm \text { SD } \\
\end{array}$ & $\begin{array}{c}\text { Low Syntax } \\
\text { Score Group } \\
\mathbf{N}=\mathbf{1 0 0} \\
\text { Mean } \pm \text { SD } \\
\end{array}$ & $P$ value \\
\hline \multicolumn{2}{|c|}{$\begin{array}{c}\text { Age } \\
\text { Range }(39-71)\end{array}$} & $55.81 \pm 7.87$ & $54.16 \pm 7.79$ & 0.138 \\
\hline \multirow[t]{2}{*}{ Sex } & $\begin{array}{cc}\text { Male } & (\mathrm{n}) \\
& (\%)\end{array}$ & $\begin{array}{r}61 / 100 \\
(61 \%)\end{array}$ & $\begin{array}{l}60 / 100 \\
(60 \%)\end{array}$ & \multirow[t]{2}{*}{0.885} \\
\hline & $\begin{array}{ll}\text { Female } & (\mathrm{n}) \\
& (\%)\end{array}$ & $\begin{array}{r}39 / 100 \\
(39 \%)\end{array}$ & $\begin{array}{r}40 / 100 \\
(40 \%)\end{array}$ & \\
\hline \multirow[t]{2}{*}{ Hypertension } & $\begin{array}{ll}\text { HTN } & (\mathrm{n}) \\
& (\%) \\
\end{array}$ & $\begin{array}{r}54 / 100 \\
(54 \%)\end{array}$ & $\begin{array}{r}56 / 100 \\
(56 \%)\end{array}$ & \multirow[t]{2}{*}{0.69} \\
\hline & $\begin{array}{ll}\text { Non }- \text { HTN } & (\mathrm{n}) \\
& (\%) \\
\end{array}$ & $\begin{array}{r}46 / 100 \\
(46 \%) \\
\end{array}$ & $\begin{array}{r}44 / 100 \\
(44 \%) \\
\end{array}$ & \\
\hline \multirow[t]{2}{*}{$\begin{array}{l}\text { Diabetes } \\
\text { mellitus }\end{array}$} & $\begin{array}{l}(\mathrm{n}) \\
(\%)\end{array}$ & $\begin{array}{r}45 / 100 \\
(45 \%) \\
\end{array}$ & $\begin{array}{r}35 / 100 \\
(35 \%) \\
\end{array}$ & \multirow[t]{2}{*}{0.165} \\
\hline & Non - DM & $\begin{array}{r}55 / 100 \\
(55 \%)\end{array}$ & $\begin{array}{r}64 / 100 \\
(64 \%)\end{array}$ & \\
\hline \multirow[t]{2}{*}{ Smoking } & Smoker & $\begin{array}{r}57 / 100 \\
(57 \%)\end{array}$ & $\begin{array}{l}52 / 100 \\
(52 \%)\end{array}$ & \multirow[t]{2}{*}{0.477} \\
\hline & $\begin{array}{r}\text { Non }- \text { Smoker (n) } \\
(\%)\end{array}$ & $\begin{array}{r}43 / 100 \\
(43 \%)\end{array}$ & $\begin{array}{l}48 / 100 \\
(48 \%)\end{array}$ & \\
\hline
\end{tabular}

Table 2: Laboratory and echocardiographic character of two groups

\begin{tabular}{|c|c|c|c|}
\hline variables & Low sx group & Mid-high sx group & P value \\
\hline creatinine & $1.06 \pm 0.2$ & $1.1 \pm 0.2$ & 0.031 \\
\hline HS-CRP & $2.37 \pm 0.66$ & $4.61 \pm 1.54$ & $\mathbf{0 . 0 0 0 1} *$ \\
\hline Troponin I & $1 \pm 0.32$ & $1.15 \pm 0.29$ & $\mathbf{0 . 4 0 8 9}$ \\
\hline LVEF & $50 \pm 8$ & $41 \pm 9$ & $<\mathbf{0 . 0 0 1}$ \\
\hline
\end{tabular}

*: Statistically significant at $\mathrm{p} \leq 0.05$

Table 3: Angiographic characters and clinical presentation and syntax score level in both groups

\begin{tabular}{|c|c|c|c|c|}
\hline VARIABLES & & Low sx group & Mid-high sx group & P value \\
\hline \multirow{2}{*}{ Presentation } & STE-ACS & $(56 \%)$ & $(61 \%)$ & \multirow[t]{2}{*}{0.47} \\
\hline & NSTE-ACS & $44 \quad(44 \%)$ & (39\%) & \\
\hline Syntax Score & & $17.79 \pm 3.19$ & $27.19 \pm 4.49$ & 0.0001 \\
\hline \multirow{3}{*}{$\begin{array}{c}\text { Number } \\
\text { of diseased } \\
\text { vessels }\end{array}$} & single & $55 \quad(55 \%)$ & $26 \quad(26 \%)$ & \multirow{3}{*}{$<0.001$} \\
\hline & two & $(31 \%)$ & $(33 \%)$ & \\
\hline & three & $(14 \%)$ & $(41 \%)$ & \\
\hline \multirow{3}{*}{$\begin{array}{l}\text { Culprit } \\
\text { coronary } \\
\text { artery }\end{array}$} & LAD & $(40 \%)$ & $(64 \%)$ & \multirow{3}{*}{$<0.001$} \\
\hline & LCX & $(26 \%)$ & $(8 \%)$ & \\
\hline & RCA & $(34 \%)$ & $(28 \%)$ & \\
\hline
\end{tabular}

Table 4: Correlation between HS-CRP and Syntax Score in two groups

\begin{tabular}{|l|c|c|}
\hline Variables & \multicolumn{2}{|c|}{ HS-CRP } \\
\cline { 2 - 3 } & r & P \\
\hline Syntax Score & 0.567 & $\mathbf{0 . 0 0 0 1} *$ \\
\hline
\end{tabular}

*: Statistically significant at $\mathrm{p} \leq 0.05$ 
Abdelaziz Rezk et al.

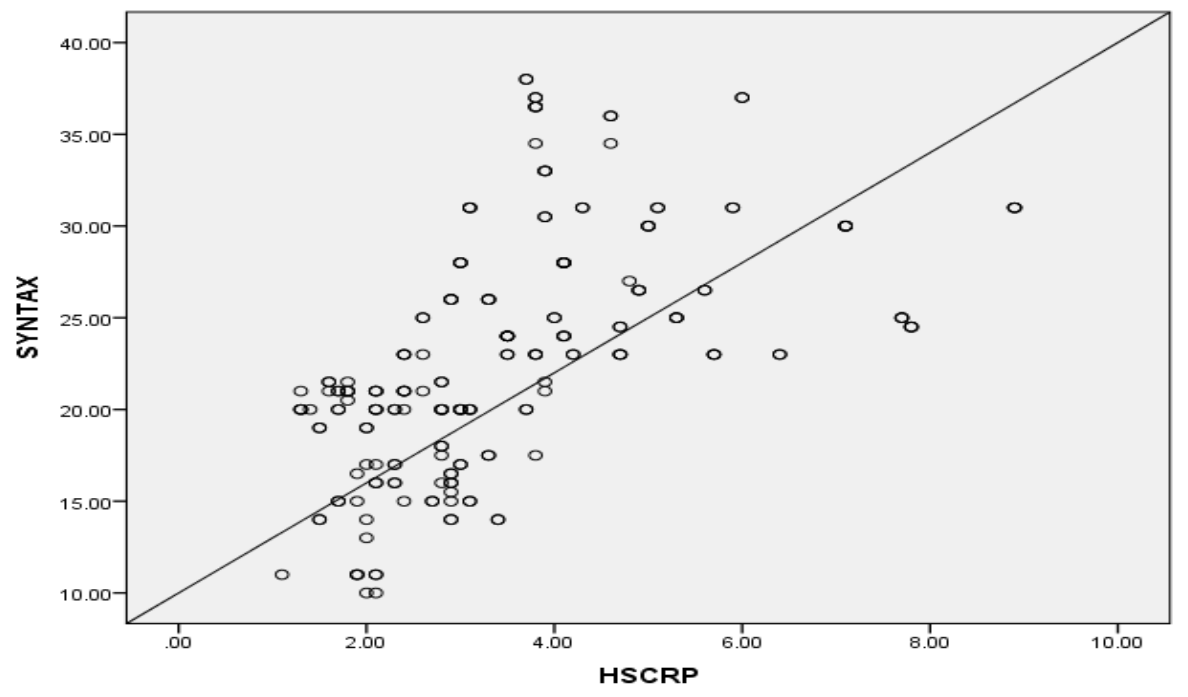

Figure 1: Correlation between syntax score and levels of HS-CRP

Table 5: Regression Linear Curve of HS-CRP as a marker and predictor of coronary artery disease complexity in studied groups.

\begin{tabular}{|l|l|l|l|l|}
\hline Predictor & R & R square & F & P value \\
\hline HS-CRP & $\mathbf{0 . 5 6 3}$ & $\mathbf{0 . 3 1 6}$ & $\mathbf{9 1 . 4 4 \%}$ & $\mathbf{0 . 0 0 0 1 *}$ \\
\hline
\end{tabular}

*: Statistically significant at $\mathrm{p} \leq 0.05$

Univariate logistic regression analysis was used to investigate the significance of hs-crp as independent predictors of high SXScore in ACS patients. This analysis showed that increased serum hs-CRP levels is strong predictors of a high SXScore.

Table 6: ROC Curve of HS-CRP as a marker and predictor of coronary artery disease complexity in studied groups

\begin{tabular}{|l|l|l|l|l|}
\hline Variable & CUT-OFF & SP & SN & ACC \\
\hline HS-CRP & $\geq 2.5$ & $\mathbf{4 1 \%}$ & $\mathbf{9 7 \%}$ & $\mathbf{0 . 9 4}$ \\
\hline
\end{tabular}

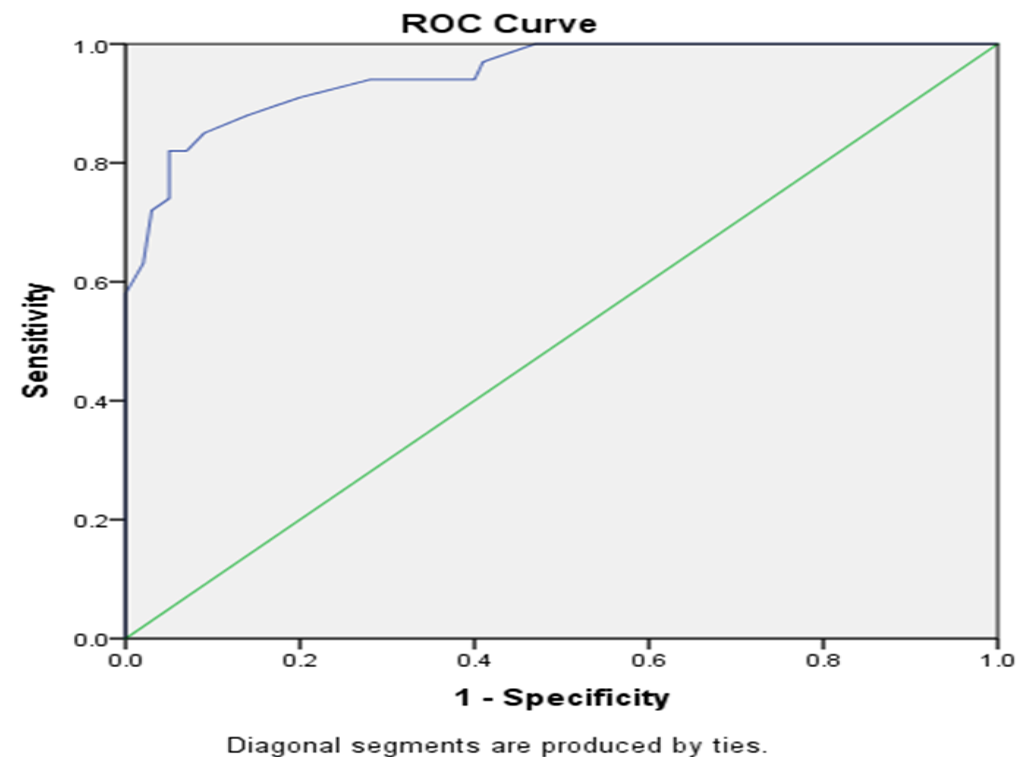

Figure 2: (ROC) curve analysis for Hs-CRP levels in predicting patients with a high Syntax Score 


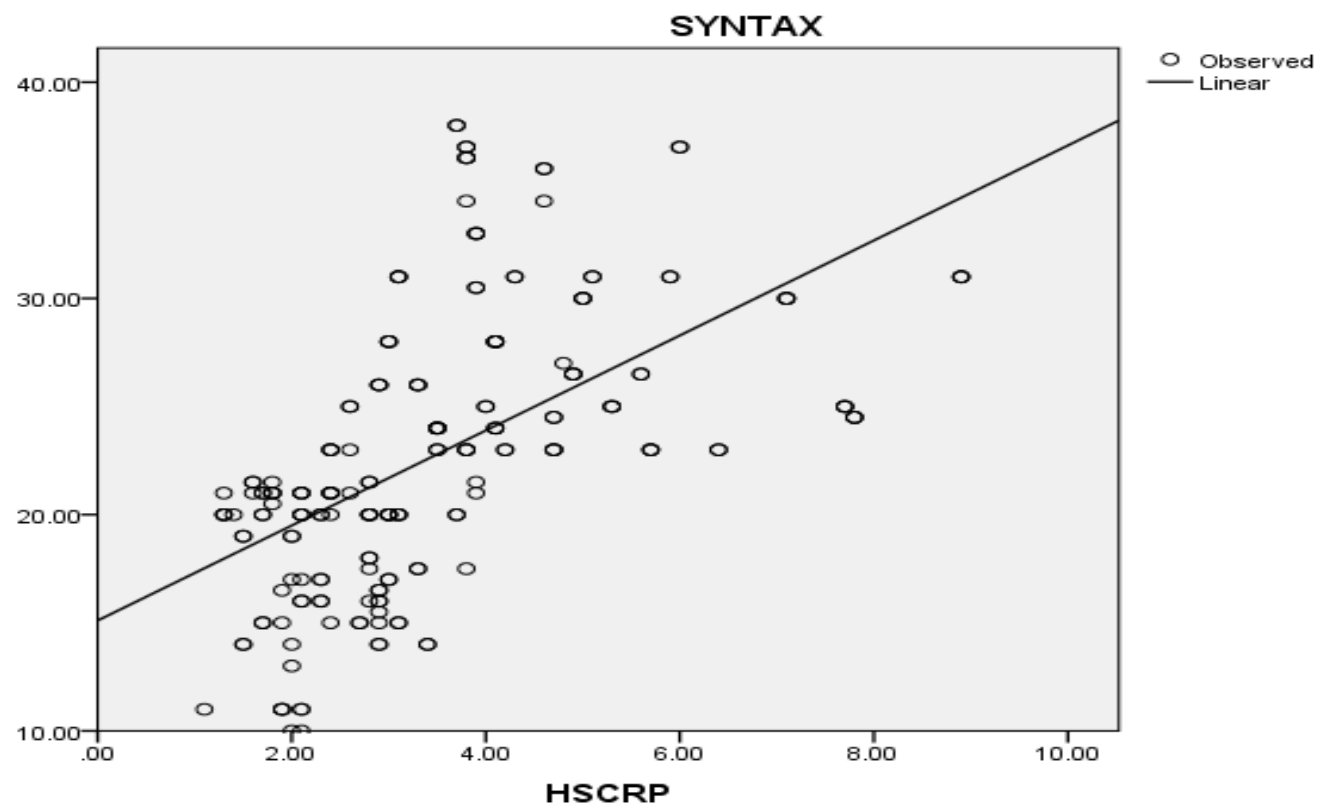

Figure 3: Regression Linear Curve of HS-CRP as a marker and predictor of coronary artery disease complexity in studied groups.

\section{DISCUSSION}

Regarding patients diagnosed with ACS, several clinical and laboratory parameters and risk scores have been proven to be of great value for short and longterm risk stratification ${ }^{(14)}$.

However, despite these available parameters and scores, there are still insufficiencies in determining the proper risk assessment of patients with ACS. Many biomarkers, especially chronic inflammation markers, have been studied for determining the risk assessment of ACS patients.

The correlation between C-reactive protein and coronary atherosclerosis is not fully understood. This study showed a positive association between hs-CRP and severity of CAD by SXScore in patients with ACS.

Many previous reports showed that there is a close relationship between the severity of atherosclerosis and serum levels of CRP.

The importance of this study comes from fact that it is one of few studies used SX score as quantitative tool for severity of $\mathrm{CAD}^{(15)}$. CRP measurement has a lot of advantages. Firstly, it is a stable compound and secondly, it can be measured at any time of the day without regards to the biological clock. In contrast to results of cytokine measurements, such as IL-6, no circadian variation appears to exist for hs-CRP. Thus, clinical testing for hs-CRP can be accomplished without regard for the time of day $(16,17)$.

Based on what were showed in the Uppsala Longtitudinal Study of Adult Men (ULSAM) that hsCRP was an independent predictor of major fatal cardiovascular events and total mortality even in the presence of other important biomarkers such as troponin I and N-terminal pro-brain natriuretic peptide $(\mathrm{NT}-\text { proBNP) })^{(\mathbf{1 7 )}}$.

The Emerging Risk Factors Collaboration study reported in a meta-analysis that hs-CRP was an independent predictor of major vascular outcomes in predicting future events and hs-

CRP performed equally well or better than several traditional risk factors ${ }^{(18)}$.

Bogaty et al. ${ }^{(19)}$ reported that assessment of severity of atherosclerosis through using only the Gensini score as grading tool showed that hsCRP levels correlated with the severity of atherosclerosis ${ }^{(19)}$.

In this study, we used the SXScore (which was more sensitive than the Gensini score) to assess the severity of atherosclerosis.

In our study we observed that there were no significant differences between low syntax group and high-mid syntax groups regarding, (Age, sex, diabetes, hypertension, smoking, serum creatinine, serum level of high sensitive troponin and clinical presentation). Benedek et al. reported similar results ${ }^{(20)}$.

LVEF was significantly lower in the patients with an intermediate-high SXScore compared to low SXScore tertiles $(\mathrm{P}<0.001)$ This comes into agreement with Choudhary et al. ${ }^{(21)}$ as they concluded that, patients with high syntax score had greater systolic dysfunction (lower LVEF) and, as they had greater area of myocardium involvement, which may partly explain bad outcome in this group. In this study subjects with a lower SYNTAX score had significantly fewer multiple-vessel lesions. These results were agreed with study performed by Cho et al. (22) on overall, 396 patients where they found that patients with a lower syntax score had significantly fewer multiplevessel lesions. 
Our team investigated the relationship between the SXScore and hsCRP value when they were analyzed with regression analysis. According to the univariate regression analysis in table 5 , the coefficient of correlation (r) of hsCRP was $\mathbf{0 . 5 6 3}$, while coefficient of determination (R Square) was $\mathbf{0 . 3 1 6}$ with efficacy of this test (f) was $\mathbf{9 1 . 4 4 \%}$, and the $\mathrm{p}$ value for this test was found to be statistically significant that. We could confirm that hsCRP was a strong predictive value for the SXScore. We believe it is important to demonstrate that the hsCRP level is a predictive indicator not only for an intermediate and high SXScore $(\geqq 22)$, but also for the SXScore value itself.

Careful assessment of our data revealed that there is significant difference in culprit coronary lesion between two groups where, In low sx group: (40\%) of patients had (LAD) as their culprit lesion while (26\%) of patients had (LCX) as their culprit lesion, and (34\%) of patients had (RCA) as culprit lesion. In highmid sx group: (64\%) of patients had (LAD) as their culprit lesion while (8\%) of patients had (LCX) as their culprit lesion, and 28 patients (28\%) had (RCA) as culprit lesion.

Taking in consideration that this result supports the analysis of database from, Acute Catheterisation and Urgent Intervention Triage StrategY (ACUITY) study which included 2,255 patients with MV disease who underwent coronary angio and intervention in the setting of ACS (23). When using more complex statistical analysis for our results it showed that hsCRP at a cut-off point of $\geq 2.5 \mathrm{mg} / \mathrm{L}$ was highly sensitive (97\%) and specific (41\%) and was a strongly independent predictor of a high SXScore in patients with ACS. where accuracy of test was $(\mathrm{acc}=0.94)$. This comes into agreement with data published by Karadeniz et al. ${ }^{(24)}$, in which total of 321 of ACS patients underwent coronary angiography; the result was similar to our result where they found that hs-CRP is strong independent predictor of sx score.

\section{Study limitations:}

The assessment of coronary angiographic findings was limited to visual interpretation and angiography is a technique that detects only major coronary arterial lesions. There is variation in age, diabetes, hypertension in the low sx group than in the high-mid group. However, these differences do not reach a statistical significance; most probably due to the small number of the patients of the study.

\section{CONCLUSION}

We concluded that as regard the category of patients who suffer from ACS and have higher levels of serum hs-CRP, higher levels of syntax score and consequently more complex and severe atherosclerotic lesions could be expected.

\section{REFERENCES}

1. Sianos G, Morel MA, Kappetein AP et al. (2005): The SYNTAX Score: an angiographic tool grading the complexity of coronary artery disease. EuroIntervention, 1: 219-27.

2. Serruys PW, Onuma Y, Garg $S$ et al. (2009): Assessment of the SYNTAX score in the Syntax study. EuroIntervention , 5: 50-6.

3. SYNTAX Working Group (2015): SYNTAX score calculator. Available at: http://www.syntaxscore.com. 4. Palmerini T, Genereux P, Caixeta A et al. (2011): Prognostic value of the SYNTAX score in patients with acute coronary syndromes undergoing percutaneous coronary intervention: analysis from the ACUITY (Acute Catheterization and Urgent Intervention Triage StrategY) trial. J Am Coll Cardiol., 57: 2389-97.

5. Valgimigli $M$, Serruys $P W$, Tsuchida $K$ et al. (2007): Cyphering the complexityof coronary artery disease using the syntax score to predict clinical outcome in patients with three-vessel lumen obstruction undergoing percutaneous coronary intervention. Am J Cardiol., 99: 1072-81.

6. Capodanno D, Di Salvo ME, Cincotta G et al. (2009): Usefulness of the SYNTAX score for predicting clinical outcome after percutaneous coronary intervention of unprotected left main coronary artery disease. Circ Cardiovasc Interv., 2: 302-8.

7. Huang G, Zhao JL, Du $\mathrm{H}$ et al. (2010): Coronary score adds prognostic information for patients with acute coronary syndrome. Circ J., 74: 490-5.

8. van Gaal WJ, Ponnuthurai FA, Selvanayagam J et al. (2009): The Syntax score predicts peri-procedural myocardial necrosis during percutaneous coronary intervention. Int J Cardiol., 135: 60-5.

9. Halkin A, Singh M, Nikolsky E et al. (2005): Prediction of mortality after primary percutaneous coronary intervention for acute myocardial infarction: the CADILLAC risk score. J Am Coll Cardiol., 45: 1397-405.

10. Yan AT, Yan RT, Tan $M$ et al.(2005): In-hospital revascularization and one-year outcome of acute coronary syndrome patients stratified by the GRACE risk score. Am J Cardiol ., 96: 913-6.

11. de Lemos JA, McGuire DK, Drazner MH (2003): B-type natriuretic peptide in cardiovascular disease. Lancet, 362: 316-22.

12. Weber M, Dill T, Arnold R et al. (2004): N-terminal B-type natriuretic peptide predicts extent of coronary artery disease and ischemia in patients with stable angina pectoris. Am Heart J., 148: 612-20.

13. Cockcroft DW, Gault MH (1976): Prediction of creatinine clearance from serum creatinine. Nephron, 16: 31-41.

14. Koenig W (2013): High-sensitivity C-reactive protein and atherosclerotic disease: from improved risk prediction to risk-guided therapy. Int J Cardiol., 168: 5126-34.

15. Zethelius B, Berglund L, Sundstrom J et al. (2008): Use of multiple biomarkers to improve the prediction 
of death fromcardiovascular causes. N Engl J Med., 358: $2107-16$

16. Ridker PM, Brown NJ, Vaughan DE et al. (2004): Established and emerging plasma biomarkers in the prediction of first atherothrombotic events. Circulation, 109: 6-19.

17. Haverkate F, Thompson SG, Pyke SD et al. (1997): Production of C-reactive protein and risk of coronary events in stable and unstable angina. Lancet, 349: 4626.

18. Ross $R$ (1999): Atherosclerosis--an inflammatory disease. N Engl J Med., 340: 115-26.

19. Bogaty P, Boyer L, Simard S et al. (2008): Clinical utility of C-reactive protein measured at admission, hospital discharge, and 1 month later to predict outcome in patients with acute coronary disease. The RISCA (recurrence and inflammation in the 2339acute coronary syndromes) study. J Am Coll Cardiol., 51: 46.

20- Benedek T, Jako B, Suciu $Z$ et al. (2014): Correlations between severity of coronary atherosclerosis and persistent elevation of circulating
C-reactive protein levels 30 days after an acute myocardial infarction, Revista Romana de Medicina de Laborator., 22(1), 49-61.

21. Choudhary S (2017): Association of syntax score with short-term outcomes among acute ST-elevation myocardial infarction patients undergoing primary PCI. Indian Heart Journal, 69: 20-23.

22- Cho Y, Shimura S, Aki A et al. (2016): The SYNTAX score is correlated with long-term outcomes of coronary artery bypass grafting for complex coronary artery lesions. Interactive Cardiovascular and Thoracic Surgery, 23(1): 125-132.

23. Chang M, Lee CW, Ahn JM et al. (2017): Comparison of Outcome of Coronary Artery Bypass Grafting Versus Drug-Eluting Stent Implantation for Non-ST-Elevation Acute Coronary Syndrome. The American Journal of Cardiology, 120(3), 380-386.

24. Karadeniz M, Duran M, Akyel A et al. (2015): High sensitive CRP level is associated with intermediate and high syntax score in patients with acute coronary syndrome. International Heart Journal, 56(4): 377-380. 